\title{
The chromosome copy number of the hyperthermophilic archaeon Thermococcus kodakarensis KOD1
}

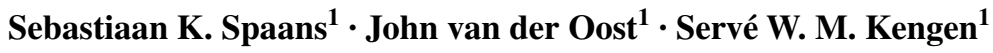

Received: 26 February 2015 / Accepted: 12 April 2015 / Published online: 8 May 2015

(C) The Author(s) 2015. This article is published with open access at Springerlink.com

\begin{abstract}
The euryarchaeon Thermococcus kodakarensis is a well-characterized anaerobic hyperthermophilic heterotroph and due to the availability of genetic engineering systems it has become one of the model organisms for studying Archaea. Despite this prominent role among the Euryarchaeota, no data about the ploidy level of this species is available. While polyploidy has been shown to exist in various Euryarchaeota, especially Halobacteria, the chromosome copy number of species belonging to one of the major orders within that phylum, i.e., the Thermococcales (including Thermococcus spp. and Pyrococcus spp.), has never been determined. This prompted us to investigate the chromosome copy number of T. kodakarensis. In this study, we demonstrate that $T$. kodakarensis is polyploid with a chromosome copy number that varies between 7 and 19 copies, depending on the growth phase. An apparent correlation between the presence of histones and polyploidy in Archaea is observed.
\end{abstract}

Keywords Thermococcus kodakarensis - Chromosome copy number - Genome copy number · Archaea .

Polyploidy · Euryarcheaota

Communicated by H. Atomi.

Electronic supplementary material The online version of this article (doi:10.1007/s00792-015-0750-5) contains supplementary material, which is available to authorized users.

Servé W. M. Kengen

serve.kengen@wur.nl

Sebastiaan K. Spaans

sebastiaan.spaans@wur.nl

1 Laboratory of Microbiology, Wageningen University, PO Box 8033, 6700 EJ Wageningen, The Netherlands

\section{Introduction}

Thermococcus kodakarensis is a marine hyperthermophilic, heterotrophic, obligate anaerobic euryarchaeon that can grow at temperatures ranging from 60 to $100{ }^{\circ} \mathrm{C}$, with an optimum at $85{ }^{\circ} \mathrm{C}$. T. kodakarensis can grow on a variety of organic substrates in the presence of elemental sulfur, producing hydrogen sulfide, or when pyruvate or starch are present, in the absence of elemental sulfur producing hydrogen. Under optimal conditions, T. kodakarensis grows rapidly to high cell densities, having an estimated doubling time of $\sim 40$ min. Moreover, it can be grown on solid media, forming defined but small colonies (Atomi et al. 2004; Morikawa et al. 1994). With its genome being fully sequenced (Fukui et al. 2005), its natural competence and the establishment of various genetic engineering techniques (Hileman and Santangelo 2012; Santangelo and Reeve 2011; Sato et al. 2003, 2005), T. kodakarensis rapidly became one of the model organisms for studying anaerobic Archaea.

While genome modifications are generally relatively easily obtained in this organism, and thus routinely applied [e.g., (Fukuda et al. 2008; Imanaka et al. 2006; Kanai et al. 2011; Santangelo et al. 2011; Sato et al. 2003, 2004; Takemasa et al. 2011; Yokooji et al. 2013)], occasionally, we experienced difficulties in obtaining transformants with two homologous recombinations. Without going into too much detail, those data suggested that the desired double crossover modification co-existed with single crossover insertions, as well as with the wild-type chromosome. The difficulty in obtaining homozygous recombinant cultures by repetitive cycles of re-streaking and screening, as well as the repeated observation of this phenomenon among different transformation attempts, suggested that $T$. kodakarensis may possess multiple copies of its chromosome. 
In contrast to the general idea of prokaryotes being monoploid, polyploidy has in recent years been demonstrated for various species including many Euryarchaeota (Table 1), the phylum that $T$. kodakarensis belongs to. Although monoploid and polyploid species can co-exist within one phylum (Pecoraro et al. 2011), none of the Euryarchaeota investigated so far was monoploid. It has therefore been suggested that polyploidy might be a common feature in Euryarchaeota (Hildenbrand et al. 2011). In this study, we determined the chromosome copy number of the euryarchaeon T. kodakarensis. The copy number was determined for three different growth phases; the early exponential phase, the late exponential/linear phase and the early stationary phase. Our results confirm that T. kodakarensis is truly polyploid and that the exact ploidy level is dependent on the growth phase. In addition, a potential correlation between the presence of histones and polyploidy in Archaea is suggested.

\section{Materials and methods}

\section{Culture conditions and growth analysis}

The hyperthermophilic archaeon Thermococcus kodakarensis KOD1 wild-type strain was grown anaerobically at $85{ }^{\circ} \mathrm{C}$ in ASW-YT-Pyr medium. The ASW-YT medium was composed of $0.8 \times$ artificial seawater $(0.8 \times$ ASW), $5.0 \mathrm{~g} / \mathrm{L}$ yeast extract, $5.0 \mathrm{~g} / \mathrm{L}$ tryptone and $0.8 \mathrm{mg} / \mathrm{L}$ resazurine. Before inoculation, $5.0 \mathrm{~g} / \mathrm{L}$ sodium pyruvate (ASWYT-Pyr medium) was added to the medium, as well as $\mathrm{Na}_{2} \mathrm{~S} .9 \mathrm{H}_{2} \mathrm{O}$, until it became colorless. For all cultivations, 120 -mL serum bottles containing $40 \mathrm{~mL}$ culture with $\mathrm{N}_{2}$ as headspace were used. Cultures were continuously shaken (120 rpm) during growth. Growth was monitored by measuring the optical density at $600 \mathrm{~nm}\left(\mathrm{OD}_{600}\right)$ using a U-1500 spectrophotometer (Hitachi). Five independent cultures were grown, and their average $\mathrm{OD}_{600}$ value and their variances were calculated (Fig. 1).

\section{Sample extraction, cell lysis and determination of cell number}

Cultures for chromosome copy number determination were inoculated from fresh pre-cultures in the late exponential growth phase (1\% inoculum) and grown to the desired optical density. For each growth phase, samples were taken from three independent cultures. When the cultures reached the desired optical density, 1.0 and $1.5 \mathrm{~mL}$ samples were taken by syringe and cells were harvested by centrifugation $(5000 \mathrm{~g}, 30 \mathrm{~min}, \mathrm{RT})$, for the "agarose gel method" and "real-time PCR method", respectively. The supernatant was checked microscopically to verify that it was free of cells.
Cell pellets were stored at $-20{ }^{\circ} \mathrm{C}$ until further processing. The cell number of the cultures at the moment of sampling was determined by directly counting the cells using a Neubauer counting chamber. The pellets were resuspended in $100 \mu \mathrm{L}$ TE buffer (10 mM Tris; $0.1 \mathrm{mM}$ EDTA, pH 8.5). Cell lysis was performed by adding SDS to a final concentration of $0.3 \%$. Complete cell lysis was verified microscopically and the integrity of genomic DNA was verified by agarose gel electrophoresis.

\section{Preliminary quantification of chromosome copy number by "agarose gel method"}

To determine if $T$. kodakarensis could indeed possess multiple chromosomes per cell, and hence if it would be worth pursuing quantification by a more accurate method, a preliminary quantification experiment was performed first. This was done by preparing cell lysates of three independent cultures having an $\mathrm{OD}_{600}$ of $\sim 0.76$ (see above). To minimize the disturbing effect of SDS during agarose gel electrophoresis, the cell lysate was diluted tenfold by adding TE buffer to a final volume of $1 \mathrm{~mL}$. Aliquots of $5 \mu \mathrm{L}$ of each sample were loaded, in duplicate, on a $1 \%$ agarose gel containing the SYBR Safe DNA gel stain (Life technologies), flanked by a known amount of a $1 \mathrm{~kb}$ GeneRuler DNA ladder (Thermo scientific). Since the SYBR Safe DNA gel stain moves away from the migrating DNA, the stain was also added to the running buffer to ensure an equal concentration of the stain in the entire gel. After electrophoresis, the DNA concentration of each sample was quantified by measuring the fluorescence intensity of the corresponding bands using a G:box imager and GeneSys analysis software (Syngene) and comparing it to the ladder with known concentrations of DNA. The molecular weight (in Daltons) of one chromosome copy was computed with the "DNA molecular weight calculator" (http://www.currentprotocols.com/WileyCDA), which was then converted to its mass (in grams) using a "Dalton to grams conversion calculator" (http://www.unitconversion.org). Next, the chromosome copy number in the cell lysate was calculated by dividing the DNA concentration of the lysate by the mass of one chromosome. Finally, the number of chromosome copies per cell was calculated by combining this with the original cell density of the sample (supplementary file).

\section{Quantification of chromosome copy number by "real-time PCR method"}

To determine chromosome copy numbers, a previously developed real-time PCR approach was used (Breuert et al. 2006). A schematic overview of the method can be found in Hildenbrand et al. (2011). First, to make a standard curve, a $1-\mathrm{kb}$ fragment was amplified using conventional PCR with 
Table 1 Chromosome copy number of archaeal species and the distribution of archaeal histones

\begin{tabular}{|c|c|c|c|c|c|c|}
\hline \multirow{2}{*}{$\begin{array}{l}\text { Phylum } \\
\text { Order } \\
\text { Species }\end{array}$} & \multirow[t]{2}{*}{ Doubling time $(\mathrm{h})$} & \multicolumn{2}{|c|}{ Genome copy number } & \multirow[t]{2}{*}{ Ploidy } & \multirow[t]{2}{*}{ Histones } & \multirow[t]{2}{*}{ Reference } \\
\hline & & Exp. phase & Stat. phase & & & \\
\hline \multicolumn{7}{|l|}{ Euryarchaeota } \\
\hline \multicolumn{7}{|l|}{ Halobacteriales } \\
\hline Halobacterium cutirubrum & 6.7 & 10.6 & & Polyploid & Yes $^{\mathrm{a}}$ & Chant et al. (1986) \\
\hline Halobacterium cutirubrum & 13.3 & 6.3 & & Polyploid & Yes $^{\mathrm{a}}$ & Chant et al. (1986) \\
\hline Halobacterium salinarum & 4 & 25 & 1 & Polyploid & Yes & Breuert et al. (2006) \\
\hline Halobacterium salinarum & 8 & 25 & 15 & Polyploid & Yes & Breuert et al. (2006) \\
\hline Haloferax volcanii & 4 & 17 & 10 & Polyploid & Yes & Breuert et al. (2006) \\
\hline Haloferax mediterranei & & $30-55$ & $20-30$ & Polyploid & Yes & Liu et al. (2013) \\
\hline \multicolumn{7}{|l|}{ Methanosarcinales } \\
\hline Methanosarcina acetivorans & 6 & 18 & 16 & Polyploid & Yes & Hildenbrand et al. (2011) \\
\hline Methanosarcina acetivorans & 49 & 3 & 5 & Polyploid & Yes & Hildenbrand et al. (2011) \\
\hline \multicolumn{7}{|l|}{ Methanobacteriales } \\
\hline $\begin{array}{c}\text { Methanothermobacter } \\
\text { thermautotrophicus }\end{array}$ & & 2 & $1-2$ & Diploid $^{\mathrm{b}}$ & Yes & Majerník et al. (2005) \\
\hline \multicolumn{7}{|l|}{ Methanomicrobiales } \\
\hline Methanoculleus marisnigri & & & & & Yes & \\
\hline \multicolumn{7}{|l|}{ Thermococcales } \\
\hline Thermococcus kodakarensis & 1.25 & 19.4 & 7.5 & Polyploid & Yes & This study \\
\hline Pyrococcus furiosus & & Multiple & & & Yes & Malandrin et al. (1999) \\
\hline Pyrococcus abyssi & & Multiple & & & Yes & Marie et al. (1996) \\
\hline \multicolumn{7}{|l|}{ Methanococcales } \\
\hline Methanocaldococcus jannaschii & 0.5 & $10-15$ & $1-5$ & Polyploid & Yes & Malandrin et al. (1999) \\
\hline Methanococcus maripaludis & 2 & 55 & 30 & Polyploid & Yes & Hildenbrand et al. (2011) \\
\hline \multicolumn{7}{|l|}{ Thermoplasmatales } \\
\hline Thermoplasma acidophilum & & & & & $\mathrm{No}^{\mathrm{c}}$ & \\
\hline Thermoplasma volcanium & & & & & $\mathrm{No}^{\mathrm{c}}$ & \\
\hline Ferroplasma acidarmanus & & & & & $\mathrm{No}^{\mathrm{c}}$ & \\
\hline \multicolumn{7}{|l|}{ Archaeoglobales } \\
\hline Archaeoglobus fulgidus & & Multiple & & & Yes & Malandrin et al. (1999) \\
\hline \multicolumn{7}{|l|}{ Methanopyrales } \\
\hline Methanopyrus kandleri & & & & & Yes & \\
\hline \multicolumn{7}{|l|}{ Methanocellales } \\
\hline Methanocella paludicola & & & & & Yes & \\
\hline \multicolumn{7}{|l|}{ Crenarchaeota } \\
\hline \multicolumn{7}{|l|}{ Acidilobales } \\
\hline Acidianus hospitalis & & $1-2$ & 2 & Monoploid & No & Lundgren et al. (2008) \\
\hline \multicolumn{7}{|l|}{ Desulfurococcales } \\
\hline Aeropyrum pernix & 3.3 & $1-2$ & 2 & Monoploid & No & Lundgren et al. (2008) \\
\hline \multicolumn{7}{|l|}{ Fervidicoccales } \\
\hline Fervidicoccus fontis & & & & & No & \\
\hline \multicolumn{7}{|l|}{ Sulfolobales } \\
\hline Sulfolobus acidocaldarius & 3.5 & $1-2$ & 2 & Monoploid & No & Bernander and Poplawski (1997) \\
\hline Sulfolobus tokodaii & 8 & $1-2$ & 2 & Monoploid & No & Lundgren et al. (2008) \\
\hline Sulfolobus solfataricus & 7 & $1-2$ & 2 & Monoploid & No & Bernander and Poplawski (1997) \\
\hline Thermoproteales & & & & & & \\
\hline Pyrobaculum aerophilum & & $1-2$ & 2 & Monoploid & No & Lundgren et al. (2008) \\
\hline Pyrobaculum calidifontis & 3.4 & $1-2$ & 2 & Monoploid & No & Lundgren et al. (2008) \\
\hline
\end{tabular}


Table 1 continued

\begin{tabular}{|c|c|c|c|c|}
\hline \multirow{2}{*}{$\begin{array}{l}\text { Phylum } \\
\text { Order } \\
\text { Species }\end{array}$} & \multirow[t]{2}{*}{ Doubling time $(\mathrm{h})$} & Genome copy number & \multirow[t]{2}{*}{ Ploidy } & \multirow[t]{2}{*}{ Histones Reference } \\
\hline & & Exp. phase Stat. phase & & \\
\hline Thermofilum pendens & & & & Yes \\
\hline Caldivirga maquilingensis & & & & Yes \\
\hline Vulcanisaeta distributa & & & & Yes \\
\hline \multicolumn{5}{|l|}{ Thaumarchaeota } \\
\hline \multicolumn{5}{|l|}{ Cenarchaeales } \\
\hline Cenarchaeum symbiosum & & & & Yes \\
\hline \multicolumn{5}{|l|}{ Nitrosopumilales } \\
\hline Nitrosopumilus maritimus & & & & Yes \\
\hline \multicolumn{5}{|l|}{ Nitrososphaerales } \\
\hline Nitrososphaera viennensis & & & & Yes \\
\hline \multicolumn{5}{|l|}{ Korarchaeota } \\
\hline Candidatus Korarchaeum cryptofilum & & & & Yes \\
\hline \multicolumn{5}{|l|}{ Nanoarchaeota } \\
\hline Nanoarchaeum equitans & & & & Yes \\
\hline
\end{tabular}

The table gives an overview of all studies in which chromosome copy numbers of archaeal species have been determined. The table was adapted from a table presented by Hillenbrand et al. (2011), by including chromosome copy numbers of several additional species, grouping the results per order and including an extra column showing the presence or absence of archaeal histone homologs. The organisms that were not present in the histone database were additionally checked by BLAST for histone homologs. For the orders for which no ploidy level data is available, representative species, whose genome is available and could be screened for the presence of archaeal histone homologs, were randomly picked

${ }^{a}$ The species Halobacterium salinarum, Halobacterium halobium, and Halobacterium cutirubrum are so similar that they should be regarded as strains of one species named Halobacterium salinarum. H. cutirubrum is therefore, as such, not present in the histone database (Ventosa and Oren 1996)

b The cells grow in filaments; the numbers given are genome copies per cell, not per filament

c The thermoplasmatales do not encode archaeal histones, however, they do encode homologs of HU-proteins (bacterial histone-like proteins)

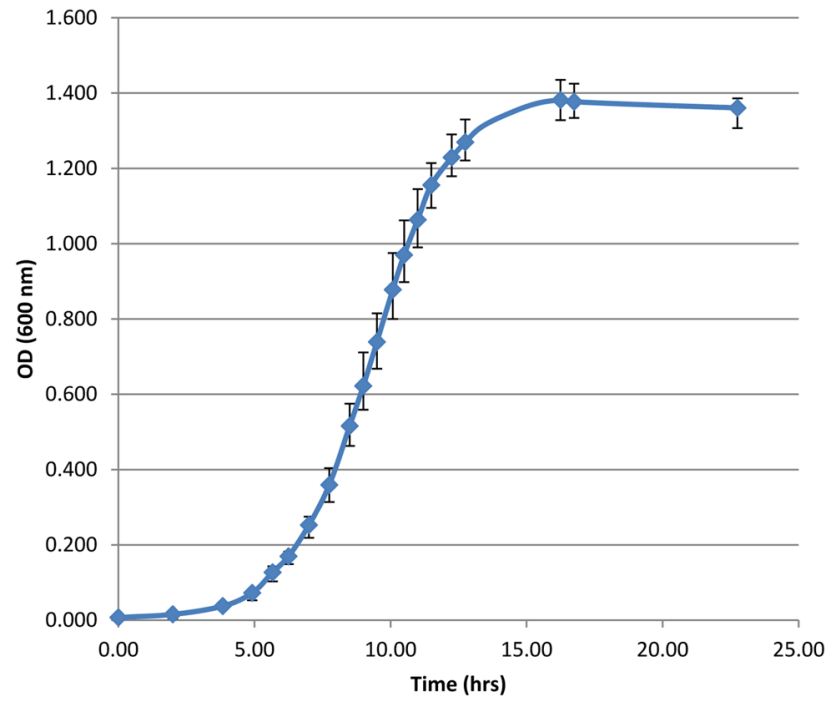

Fig. 1 Growth curve increase in optical density of Thermococcus kodakarensis KOD1 (WT) in time on ASW-YT-Pyr medium. The graph represents the average optical density at $600 \mathrm{~nm}\left(\mathrm{OD}_{600}\right)$ of five independent cultures genomic DNA as template. The genomic DNA was isolated using a genomic DNA purification kit (Thermo scientific, protocol for Gram positive bacteria). The $1 \mathrm{~kb}$ fragment generated in this study was internal to the chitinase gene (TK1765) as the amplification of this fragment had previously been shown to be successful, resulting in a clear, single product-band on gel. The primers used are shown in Table S1. The fragment was purified using preparative agarose gel electrophoresis $(0.8 \%)$ and a GeneJET Gel extraction kit (Thermo Scientific), and concentrated by an DNA Clean and Concentrator kit (ZYMO RESEARCH). The DNA concentration was determined with a Nanodrop ND-1000, and the number of DNA molecules was calculated using the molecular weight computed with "oligo calc" (http://www.basic.northwestern.edu/biotools/oligocalc.html). A serial tenfold dilution containing defined numbers of standard molecules was generated in triplicate, and used for real-time PCR analysis in parallel with a dilution series of cell lysate samples.

To minimize the inhibiting effect of SDS on real-time PCR, the cell lysate (see above) was first diluted to a final 
SDS concentration of $0.03 \%$ by adding TE buffer to a final volume of $1 \mathrm{~mL}$. This diluted cell lysate sample was used for making the dilution series that was used for realtime PCR. A 5- $\mu \mathrm{L}$ aliquot of the dilution series was used as template in the real-time PCR analyses for quantification of chromosome copy numbers (see below). To ensure that the PCR efficiency of the cell lysate dilution series and the standard dilutions series was identical, the standard dilution series was also added to 4 selected dilutions of the cell lysate sample as an internal control, yielding 6 different dilution series in total. The fragment targeted in the real-time PCR analysis was 293 bp and was internal to the standard fragment.

Real-time PCR was performed in $25-\mu \mathrm{L}$ reactions containing $5 \mu \mathrm{L}$ of template (cell lysate, standard, or cell lysate with added standard), $100 \mathrm{mM}$ of primer BG5331 and BG5332 each and $2 \times$ qPCR Master Mix (iQ SYBR Green Supermix, BIO RAD). The master mix contained antibodymediated hot start iTaq DNA polymerase, dNTPs, SYBR GREEN I, $\mathrm{MgCl}_{2}$, enhancers, stabilizers and fluorescein (concentrations not released by $\mathrm{BIO}$ RAD). The PCR reaction conditions were $10 \mathrm{~min}$ at $95^{\circ} \mathrm{C}, 40$ cycles of amplification $\left(30 \mathrm{~s}\right.$ at $95^{\circ} \mathrm{C}, 30 \mathrm{~s}$ at $59{ }^{\circ} \mathrm{C}, 30 \mathrm{~s}$ at $\left.72{ }^{\circ} \mathrm{C}\right)$, and an final incubation of $5 \mathrm{~min}$ at $72{ }^{\circ} \mathrm{C}$. The real-time PCRs were performed in a CFX96 thermal cycler (BioRad). At the end of the PCR, a melting curve was generated using the following settings: $65-95{ }^{\circ} \mathrm{C}$ with increments of $0.5{ }^{\circ} \mathrm{C} / 5 \mathrm{~s}$. For each sample the numbers of cycles was determined until its fluorescence intensity reached the threshold $(\mathrm{Cq}$ value). By comparison of the threshold cycle $(\mathrm{Cq})$ differences of the different dilutions it was verified that the PCR was exponential at least up to the threshold DNA concentration used for the analysis (i.e., a tenfold dilution corresponds to a $\mathrm{Cq}$ difference of $3.6>\mathrm{Cq}>3.1$, having an efficiency of $90-110 \%$ ) The $C q$ values of the standards were used to construct a standard curve (Fig. 3) that was used to determine the chromosome copy number in the cell lysates and to check the PCR efficiency in the cell lysates including internal standards. In combination with the cell density, the number of chromosome copies per cell was calculated.

\section{Method optimization}

The protocol as outlined above was established by optimizing different steps individually. First, various genomic DNA isolation methods, such as sonication, French press, osmotic shock, CTAB/NaCl, lysozyme, SDS or repetitive freeze-thaw cycles were tested for their effectiveness (extent of cell lysis and gDNA recovery) and ability to leave the genomic DNA intact. The only method meeting both standards, and yielding reproducible results, was the addition of SDS to the cell suspensions. Since SDS is known to have disturbing effects on both PCR and agarose gel electrophoresis, a series of SDS concentrations was tested to determine the minimal concentration needed for complete lysis and yielding reproducible results. This was found to be $0.3 \%$. Chromosome copy number quantification by the "agarose gel method" was improved by addition of the SYBR Safe DNA gel stain to the running buffer to ensure an equal concentration of the stain in the entire gel. Real-time PCR was optimized by testing three different primer sets for the amplification of a small internal fragment (all $300 \mathrm{bp}$ ). For each primer set, the optimal annealing temperature was determined by gradient PCR. The primer set BG5331-BG5332 (see Table S1), resulting in the amplification of a 293-bp fragment, turned out to be the most reproducible, without any artificial by-products. The latter was confirmed by visualizing the amplified fragments on a $1 \%$ agarose gel as well as by including a melting curve in the real-time PCR protocol.

\section{Results}

\section{Growth analysis and preliminary quantification by "agarose gel method"}

To make growth curves, five independent cultures of $T$. kodakarensis were grown on pyruvate, and their average $\mathrm{OD}_{600}$ values and their variances were used to construct Fig. 1. The results show that growth is highly reproducible, which is a prerequisite for reliable chromosome copy number quantification methods. Before an exact chromosome copy number quantification was performed, however, a preliminary in-gel quantification was applied first to determine the probability of polyploidy in T. kodakarensis, and hence if a more exact quantification method was worth applying. This was done by preparing cell lysate samples, in duplicate, of three independent cultures having an $\mathrm{OD}_{600}$ of $\sim 0.76$. Corresponding cell densities were determined and aliquots of the 6 samples obtained were loaded on an agarose gel flanked by a known amount of a $1-\mathrm{kb}$ GeneRuler DNA ladder (Thermo Scientific) as shown in Fig. 2a. The DNA concentration and chromosome copy number of each sample was quantified by measuring the fluorescence intensity of the corresponding gel bands and comparing it to the ladder containing known concentrations of DNA. Together with the original cell density of each sample, the number of chromosome copies per cell was calculated (supplementary file). The results are shown in Fig. 2b. These results clearly indicate polyploidy in T. kodakarensis, suggesting an average chromosome copy number of $17.8 \pm 3.8$. It was therefore decided to determine the chromosome copy number by a more exact quantification method as well. 


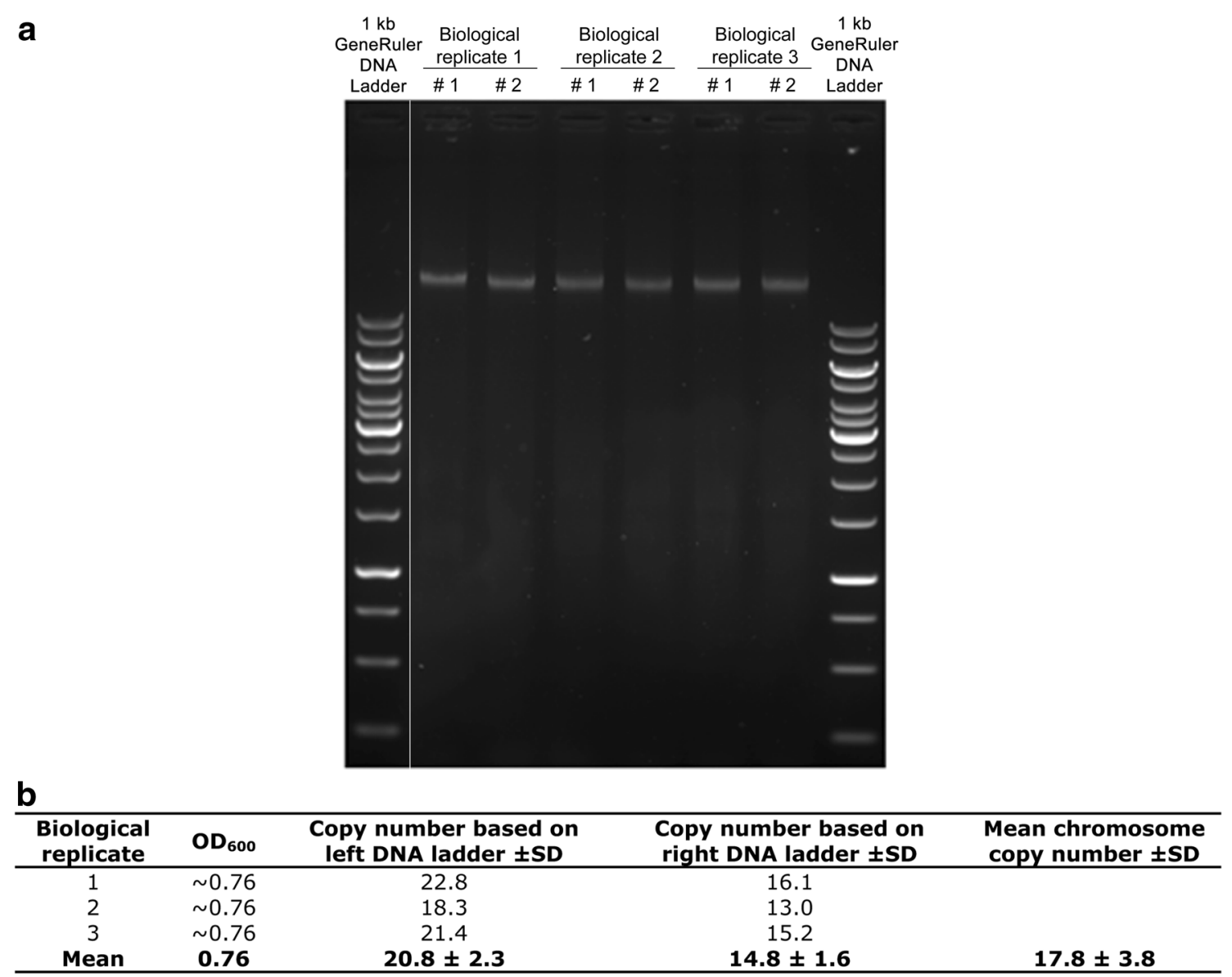

Fig. 2 Chromosome copy number quantification by "agarose gel method". a Agarose gel containing cell lysate samples of three independent cultures, in duplicate, flanked by a $1-\mathrm{kb}$ GeneRuler DNA ladder. The white line indicates non-adjacent lanes of the same gel. The DNA concentration and chromosome copy number of each sam-

\section{Quantification by "real-time PCR method"}

To certify polyploidy in T. kodakarensis and to quantify chromosome copy numbers more accurately, a previously developed real-time PCR method for quantification of chromosome copy numbers was optimized for T. kodakarensis. This chromosome quantification method has been established for haloarchaea in 2006 (Breuert et al. 2006), and has since then been applied to various other prokaryotes. The method has been validated against several independent techniques and has shown to be very reliable (Griese et al. 2011; Hildenbrand et al. 2011; Pecoraro et al. 2011). A schematic overview of the method can be found in the previous report (Hildenbrand et al. 2011).

To give a short overview, a fragment of about $1 \mathrm{~kb}$ is amplified first, using standard PCR with genomic DNA as template. A dilution series of this fragment is made and used to generate a standard curve in a real-time PCR analysis. This is done by amplifying a fragment of about ple was quantified by comparing the fluorescence intensity of the gel bands to that of the left and right ladder. $\mathbf{b}$ The number of chromosome copies per cell. This was calculated by dividing the chromosome copy number of the lysate by the original cell density of each replicate

$300 \mathrm{bp}$, internal to the standard fragment, and by plotting the threshold value $(\mathrm{Cq})$ against the corresponding template concentration (in copies/ $\mu \mathrm{L}$ ). To quantify the chromosome copy number of the species of interest, cells are lysed and a dilution series of the resulting cell lysates is analyzed by real-time PCR in parallel to the standards. By comparing the $C \mathrm{q}$ values of the cell lysate samples to the standard curve, the number of chromosome copies per sample can be determined, which in combination with the cell density of the corresponding culture, allows for calculating the ploidy level.

Cultures for chromosome copy number determination were grown to the optical densities as shown in Fig. 3, representing the early exponential phase, late exponential/ linear phase and early stationary phase. For each growth phase, samples were taken from three independent cultures. When the cultures reached the desired optical density, 1.5$\mathrm{mL}$ samples were taken and cells were harvested by centrifugation and the supernatant was checked microscopically 
Fig. 3 Chromosome copy number quantification by real-time PCR method. a Selected realtime PCR results. The fluorescent intensity curves from four standard dilutions (in red), three sample dilutions (in green), and a no-template control (NTC, in blue) are shown. The reactions containing the standard dilutions were performed in triplicate and the ones containing the samples in duplicate (these technical duplicates were in addition to the biological triplicates). The curves shown are the result of serial tenfold dilutions of the templates. In addition to the selected reactions shown here, each experiment included more standard and more sample dilutions. b Standard curve. The standard curve was created by plotting the threshold values $\left(C_{\mathrm{q}}\right)$ of the individual reactions against the corresponding template concentration. c Average chromosome copy number values and their standard variations of $T$. kodakarensis in three different growth phases. The third sample of the early stationary phase (in parentheses) could be argued not to be in the early stationary phase. Therefore, the average chromosome copy number and its standard deviation in this phase was calculated by both including (in parentheses) and excluding the third biological replicate a

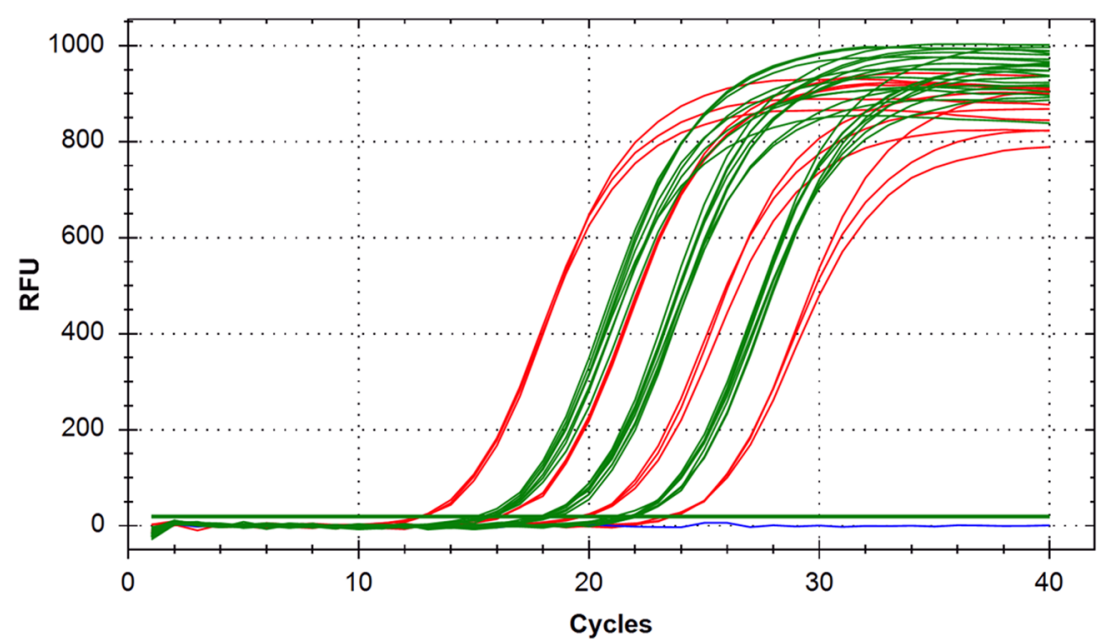

b

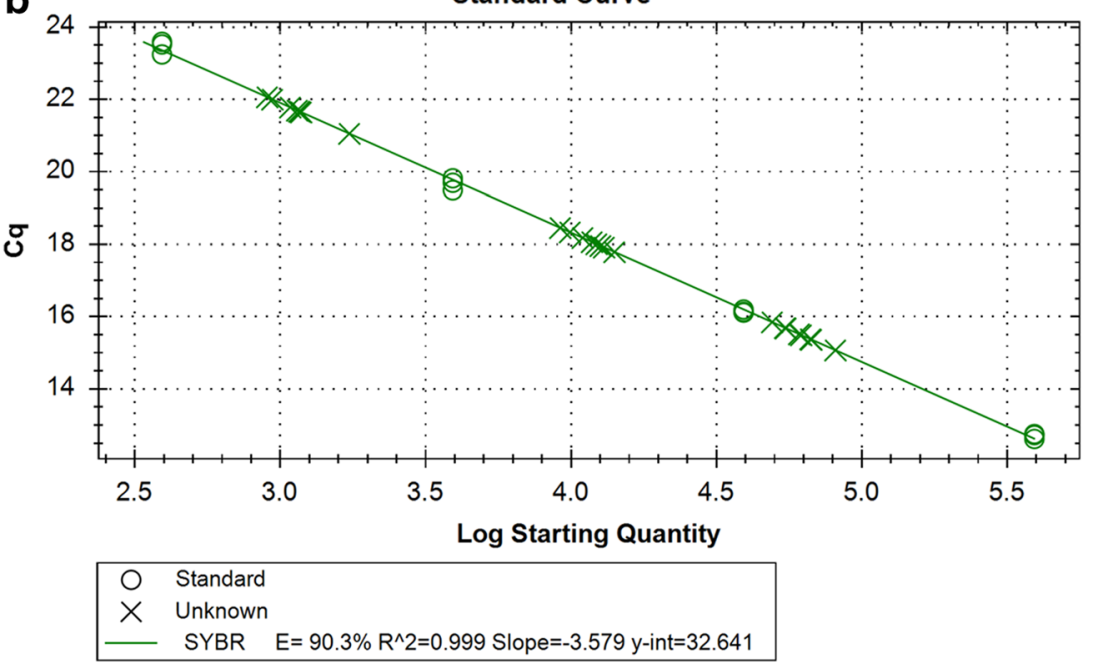

C

\begin{tabular}{cccccc}
\hline $\begin{array}{c}\text { Biological } \\
\text { replicate }\end{array}$ & Growth phase & $\mathbf{O D}_{600}$ & $\begin{array}{c}\text { Cell density } \\
\text { (cells/mI) }\end{array}$ & $\begin{array}{c}\text { Chromosome } \\
\text { copy number } \\
\pm \text { SD }\end{array}$ & $\begin{array}{c}\text { Mean } \\
\text { chromosome } \\
\text { copy } \pm \text { SD }\end{array}$ \\
\hline 1 & Early exponential & 0.375 & $1.11 \mathrm{E}+08$ & $20.5 \pm 4.9$ & \\
2 & phase & 0.397 & $1.22 \mathrm{E}+08$ & $16.9 \pm 4.9$ & $\mathbf{1 9 . 4} \pm \mathbf{4 . 9}$ \\
3 & 0.459 & $1.42 \mathrm{E}+08$ & $20.8 \pm 4.6$ & \\
\hline 1 & Late exponential / & 0.765 & $2.56 \mathrm{E}+08$ & $15.3 \pm 3.4$ & \\
2 & 0.736 & $2.33 \mathrm{E}+08$ & $12.6 \pm 2.4$ & $\mathbf{1 6 . 9} \pm \mathbf{6 . 0}$ \\
3 & Linear phase & 0.771 & $2.56 \mathrm{E}+08$ & $22.9 \pm 5.8$ & \\
\hline 1 & Early stationary & 1.43 & $8.73 \mathrm{E}+08$ & $6.9 \pm 2.5$ & $\mathbf{7 . 5} \pm \mathbf{2 . 8}$ \\
2 & phase & 1.41 & $9.30 \mathrm{E}+08$ & $8.1 \pm 3.1$ & $(8.7 \pm 3.2)$ \\
\hline 3 & $(1.33)$ & $(6.28 \mathrm{E}+08)$ & $(11.0 \pm 3.0)$ &
\end{tabular}

to verify that it was free of cells; which was the case. The cell numbers of the cultures at the moment of sampling were determined (see Fig. 3c) and cells were lysed by adding SDS, which was found to be the most effective (see "Materials and methods").

A tenfold dilution series of the cell lysates was prepared and used for real-time PCR analysis in parallel with the standard fragment. To verify that the added SDS or other components present in the cell lysate did not negatively influence the reaction itself, the standard dilution series was also added to 4 selected dilutions of the cell lysate sample as an internal control. No inhibiting effects were observed for any of the selected samples. The final real-time PCR was performed by including technical duplicates, in addition to the biological triplicates, and had an efficiency of $90.3 \%$, an $R^{2}$ of 0.999 and a slope of -3.5 . The results of the real-time PCR are shown in Fig. 3. It was found that cells in the early exponential phase contain about $19.4 \pm 4.9$ copies of their chromosome, and that this value drops to $7.5 \pm 2.8$ copies in early stationary phase. The 
two independent quantification methods thus both confirm polyploidy in T. kodakarensis.

\section{Discussion}

The results obtained in this study show that the euryarchaeon T. kodakarensis contains multiple chromosomes, with numbers varying from $\sim 7$ to 19 copies per cell. Although polyploidy has been demonstrated for several Euryarchaeota, the chromosome copy number of species belonging to one of the major orders within that phylum, i.e., the Thermococcales (including Thermococcus spp. and Pyrococcus spp.), has never been determined. The existence of polyploidy in T. kodakarensis supports the suggestion of Hildenbrand et al. (2011) that polyploidy might be a common trait of all Euryarchaeota (Hildenbrand et al. 2011). The only exception known so far is Methanothermobacter thermautotrophicus, which was reported to be diploid instead (Majerník et al. 2005). T. kodakarensis was found to have a growth-phase-dependent ploidy level having as many as $19.4 \pm 4.9$ chromosome copies at the early exponential phase, which is strongly decreasing towards the stationary phase. The observation of a growth-phasedependent ploidy level has been reported for many other prokaryotes as well, including the majority of the investigated euryarchaeal species (Breuert et al. 2006; Griese et al. 2011; Hildenbrand et al. 2011; Pecoraro et al. 2011). Unfortunately, no common pattern has emerged from the characterized prokaryotic species (Breuert et al. 2006). Where some species have a higher chromosomal copy number in the exponential phase [e.g., T. kodakarensis or some halophilic Archaea (Breuert et al. 2006)], others have a higher chromosomal copy number in the stationary phase [e.g., Azotobacter vinelandii (Maldonado et al. 1994)].

Although a chromosome copy number of around 19 might seem to be rather high, this result is comparable to copy numbers (2-55) found in many other Euryarchaeota (see Table 1). The euryarchaea Methanococcus maripaludis and Haloferax mediterranei were even found to have up to 55 chromosome copies per cell, which is the highest copy number for Archaea described so far. While this is more than double the number found for T. kodakarensis, it is still low compared to the copy numbers reported for the cyanobacterium Synechocystis sp. PCC 6803 or the firmicute Epulopiscium spp., having up to 218 or even tens of thousands of copies, respectively (Griese et al. 2011; Mendell et al. 2008). The bacterium Epulopiscium spp., however, is quite extraordinary in that it is one of the largest known bacteria $(200-700 \mu \mathrm{m})$, having sufficient intracellular space to contain an exceptionally high number of chromosomes (Angert et al. 1993). Synechocystis sp. PCC 6803, in contrast, is similar in size $(2 \mu \mathrm{m})$ to $T$. kodakarensis, and one may wonder how it is able to accommodate up to 10 times more chromosomes (Atomi et al. 2004; Lea-Smith et al. 2014).

The finding that T. kodakarensis is polyploid is also in good agreement with results obtained in chromosome quantification studies of the genetically accessible extremophiles D. radiodurans and T. thermophilus (Cao et al. 2010; Ohtani et al. 2010). Both bacteria were reported to have similar engineering difficulties as the ones occasionally observed for T. kodakarensis and were subsequently found to have multiple copies (6-10 and 4-5, respectively) of their chromosome. The existence of multiple chromosome copies can readily explain the occasionally observed difficulty in obtaining homozygous recombinant cultures, as it entails that the desired strain is only obtained when the modification is incorporated in all chromosome copies.

\section{Physical implications of polyploidy}

Based on the results obtained, several questions arise: (1) how are polyploid prokaryotes able to fit that many copies of its chromosome in a cell, and related to that, how is spontaneous aggregation due to the high DNA concentrations prevented, and (2) what possible evolutionary advantages could polyploidy have? Since the latter aspect has been thoroughly covered in recent papers (Soppa 2013; Zerulla et al. 2014; Zerulla and Soppa 2014), describing potential advantages such as conferring resistance to DNA damage or allowing heterozygosity to occur (both of which might be beneficial for survival in extreme conditions), only the former question will be briefly addressed here.

It is well known that all living things have to extensively condense their genomic DNA to fit it in the physically small space of a cell. In addition, this so-called DNA packaging also prevents spontaneous aggregation due to the high concentration imposed by the confinement (Minsky et al. 1997). The key mechanisms employed for DNA packaging are DNA supercoiling, macromolecular crowding and the association of DNA-binding proteins that fold the genome into a more condensed structure. The interactions between DNA-binding proteins and the genomic DNA can have different structural effects on DNA, such as bending, bridging or wrapping, each of which mediates DNA packaging in a different way [reviewed in e.g., (Luijsterburg et al. 2008; Sandman et al. 1998)].

DNA-binding proteins are generally small basic proteins that are abundantly present. The best examples are the eukaryotic histones that wrap genomic DNA by folding it around their surface forming so-called nucleosomes, the primary unit of DNA compaction. Archaeal homologs of histone proteins have been found in almost all Euryarchaeota (including T. kodakarensis), Nanoarchaeota and 
Thaumarchaeota, but are generally not encoded by Crenarchaeota (Čuboňová et al. 2012; Higashibata et al. 1999; Maruyama et al. 2013, 2011; Sandman and Reeve 2006; White and Bell 2002). Interestingly, a similar dichotomy between Euryarchaeota and Crenarchaeota can be observed regarding their ploidy levels: almost all tested Euryarchaeal species have been found to be polyploid, while all the Crenarchaeal species tested to date were found to be monoploid (Hildenbrand et al. 2011) (Table 1). Whether there is any correlation between these observations is currently unknown, however, it is tempting to speculate that histones play an important role in enabling polyploidy in Archaea.

While DNA packaging explains how a single genome is sufficiently condensed to fit the physically small space of a cell, it does not clarify how many chromosome copies actually fit in. To answer this question we compared the situation in T. kodakarensis with the situation in E. coli, for which it is known that on average two chromosome copies [ 9.2 Mbp in total, $\sim 0.136-0.16 \mu \mathrm{m}^{3}$ (Odijk 1998)] occupy about a quarter of the total cell volume [total cell volume of $E$. coli: 0.58-0.69 $\mu \mathrm{m}^{3}$ (Kubitschek 1990)] (Dame 2005). Assuming that DNA packaging in T. kodakarensis is as efficient as in E. coli and that the irregular cocci of T. kodakarensis are perfect spheres (having a cell diameter of $1-2 \mu \mathrm{m}$ and hence a cell volume of $\left.\sim 0.52-4.18 \mu \mathrm{m}^{3}\right)$, fitting up to $\sim 19$ copies of its chromosome $(\sim 39.7 \mathrm{Mbp}$ in total, $\sim 0.587-0.690 \mu \mathrm{m}^{3}$ ), should physically, thus, be possible.

\section{Conclusion}

We clearly showed that $T$. kodakarensis has multiple chromosome copies and that this result supports earlier reports on the presence of polyploidy in Euryarchaeota. The existence of multiple chromosome copies can also readily explain the occasionally observed difficulty in obtaining homozygous recombinant cultures, as it entails that the desired strain is only acquired when the modification is incorporated in all chromosome copies. Moreover, an apparent correlation between the presence of histones and polyploidy in Archaea is observed. The histones may assist in efficient DNA packaging which is required for fitting multiple chromosomes in a single cell.

Acknowledgments We thank Jörg Soppa from the Goethe-University for valuable advice concerning the chromosome quantification by the "real-time PCR method" and Remus Dame from Leiden University for his valuable input on discussing the physical implications of polyploidy.This work is part of the Open Programme ("Biological hydrogen production beyond current limits" file number 820.02.011), which is financed by the Netherlands Organisation for Scientific Research (NWO).
Open Access This article is distributed under the terms of the Creative Commons Attribution 4.0 International License (http://creativecommons.org/licenses/by/4.0/), which permits unrestricted use, distribution, and reproduction in any medium, provided you give appropriate credit to the original author(s) and the source, provide a link to the Creative Commons license, and indicate if changes were made.

\section{References}

Angert ER, Clements KD, Pace NR (1993) The largest bacterium. Nature 362:239-241

Atomi H, Toshiaki F, Tamotsu K, Morikawa M, Imanaka T (2004) Description of Thermococcus kodakaraensis sp. nov., a well studied hyperthermophilic archaeon previously reported as Pyrococcus sp. KOD1. Archaea 1:263-267

Bernander R, Poplawski A (1997) Cell cycle characteristics of thermophilic archaea. J Bacteriol 179:4963-4969

Breuert S, Allers T, Spohn G, Soppa J (2006) Regulated polyploidy in halophilic Archaea. PLoS One 1:e92. doi:10.1371/journal. pone.0000092

Cao Z, Mueller CW, Julin DA (2010) Analysis of the recJ gene and protein from Deinococcus radiodurans. DNA Repair (Amst) 9:66-75. doi:10.1016/j.dnarep.2009.10.009

Chant J, Hui I, De Jong-Wong D, Shimmin L, Dennis PP (1986) The protein synthesizing machinery of the archaebacterium Halobacterium cutirubrum: molecular characterization. Syst Appl Microbiol 7:106-114. doi:10.1016/S0723-2020(86)80132-1

Čuboňová L, Katano M, Kanai T, Atomi H, Reeve JN, Santangelo TJ (2012) An Archaeal histone is required for transformation of Thermococcus kodakarensis. J Bacteriol 194:6864-6874. doi:10.1128/jb.01523-12

Dame RT (2005) The role of nucleoid-associated proteins in the organization and compaction of bacterial chromatin. Mol Microbiol 56:858-870. doi:10.1111/j.1365-2958.2005.04598.x

Fukuda W, Morimoto N, Imanaka T, Fujiwara S (2008) Agmatine is essential for the cell growth of Thermococcus kodakaraensis. Fems Microbiol Lett 287:113-120. doi:10.1111/j.1574-6968.2008.01303.x

Fukui T, Atomi H, Kanai T, Matsumi R, Fujiwara S, Imanaka T (2005) Complete genome sequence of the hyperthermophilic archaeon Thermococcus kodakaraensis KOD1 and comparison with Pyrococcus genomes. Genome Res 15:352-363

Griese M, Lange C, Soppa J (2011) Ploidy in cyanobacteria. Fems Microbiol Lett 323:124-131. doi:10.1111/j.1574-6968.2011.02368.x

Higashibata H, Fujiwara S, Takagi M, Imanaka T (1999) Analysis of DNA compaction profile and intracellular contents of Archaeal histones from Pyrococcus kodakaraensis KOD1. Biochem Biophys Res Commun 258:416-424. doi:10.1006/bbrc.1999.0533

Hildenbrand C, Stock T, Lange C, Rother M, Soppa J (2011) Genome copy numbers and gene conversion in methanogenic Archaea. J Bacteriol 193:734-743. doi:10.1128/jb.01016-10

Hileman TH, Santangelo TJ (2012) Genetics techniques for Thermococcus kodakarensis. Front Microbiol 3:195

Imanaka H, Yamatsu A, Fukui T, Atomi H, Imanaka T (2006) Phosphoenolpyruvate synthase plays an essential role for glycolysis in the modified Embden-Meyerhof pathway in Thermococcus kodakarensis. Mol Microbiol 61:898-909. doi:10.1111/j.1365-2958.2006.05287.x

Kanai T, Matsuoka R, Beppu H, Nakajima A, Okada Y, Atomi H, Imanaka T (2011) Distinct physiological roles of the three [NiFe]-hydrogenase orthologs in the hyperthermophilic Archaeon Thermococcus kodakarensis. J Bacteriol 193:3109_ 3116. doi:10.1128/jb.01072-10 
Kubitschek H (1990) Cell volume increase in Escherichia coli after shifts to richer media. J Bacteriol 172:94-101

Lea-Smith DJ, Bombelli P, Dennis JS, Scott SA, Smith AG, Howe CJ (2014) Phycobilisome-deficient strains of Synechocystis sp. PCC 6803 have reduced size and require carbon-limiting conditions to exhibit enhanced productivity. Plant Physiol 165:705-714. doi:10.1104/pp.114.237206

Liu X, Miao D, Zhang F, Wu Z, Liu J, Xiang H (2013) Characterization of the minimal replicon of pHM300 and independent copy number control of major and minor chromosomes of Haloferax mediterranei. FEMS Microbiol Lett 339:66-74. doi:10.1111/1574-6968.12052

Luijsterburg MS, White MF, van Driel R, Dame RT (2008) The major architects of chromatin: architectural proteins in bacteria. Archaea Eukaryot Crit Rev Biochem Mol Biol 43:393-418. doi:10.1080/10409230802528488

Lundgren M, Malandrin L, Eriksson S, Huber H, Bernander R (2008) Cell cycle characteristics of crenarchaeota: unity among diversity. J Bacteriol 190:5362-5367. doi:10.1128/jb.00330-08

Majerník AI, Lundgren M, McDermott P, Bernander R, Chong JP (2005) DNA content and nucleoid distribution in methanothermobacter thermautotrophicus. J Bacteriol 187:1856-1858

Malandrin L, Huber H, Bernander R (1999) Nucleoid structure and partition in Methanococcus jannaschii: an archaeon with multiple copies of the chromosome. Genetics 152:1315-1323

Maldonado R, Jimenez J, Casadesus J (1994) Changes of ploidy during the Azotobacter vinelandii growth cycle. J Bacteriol 176:3911-3919

Marie D, Vaulot D, Partensky F (1996) Application of the novel nucleic acid dyes YOYO-1, YO-PRO-1, and PicoGreen for flow cytometric analysis of marine prokaryotes. Appl Environ Microbiol 62:1649-1655

Maruyama $\mathrm{H}$ et al (2011) Histone and TK0471/TrmBL2 form a novel heterogeneous genome architecture in the hyperthermophilic archaeon Thermococcus kodakarensis. Mol Biol Cell 22:386398. doi:10.1091/mbc.E10-08-0668

Maruyama $\mathrm{H}$ et al (2013) An alternative beads-on-a-string chromatin architecture in Thermococcus kodakarensis. EMBO Rep 14:711-717. doi:10.1038/embor.2013.94

Mendell JE, Clements KD, Choat JH, Angert ER (2008) Extreme polyploidy in a large bacterium. Proc Natl Acad Sci 105:6730-6734

Minsky A, Ghirlando R, Reich Z (1997) Nucleosomes: a solution to a crowded intracellular environment? J Theor Biol 188:379-385. doi:10.1006/jtbi.1997.0525

Morikawa M, Izawa Y, Rashid N, Hoaki T, Imanaka T (1994) Purification and characterization of a thermostable thiol protease from a newly isolated hyperthermophilic Pyrococcus sp. Appl Environ Microbiol 60:4559-4566

Odijk T (1998) Osmotic compaction of supercoiled DNA into a bacterial nucleoid. Biophys Chem 73:23-29. doi:10.1016/ S0301-4622(98)00115-X

Ohtani N, Tomita M, Itaya M (2010) An extreme thermophile thermus thermophilus, is a polyploid bacterium. J Bacteriol 192:54995505. doi:10.1128/JB.00662-10
Pecoraro V, Zerulla K, Lange C, Soppa J (2011) Quantification of ploidy in proteobacteria revealed the existence of monoploid, (mero-)oligoploid and polyploid species. PLoS One 6:e16392. doi:10.1371/journal.pone.0016392

Sandman K, Reeve JN (2006) Archaeal histones and the origin of the histone fold. Curr Opin Microbiol 9:520-525. doi:10.1016/j. mib.2006.08.003

Sandman K, Pereira SL, Reeve JN (1998) Diversity of prokaryotic chromosomal proteins and the origin of the nucleosome. Cell Mol Life Sci 54:1350-1364

Santangelo TJ, Reeve JN (2011) Genetic tools and manipulations of the hyperthermophilic heterotrophic archaeon Thermococcus kodakarensis. In: Extremophiles Handbook, Springer, p 567-582

Santangelo TJ, Cubonova L, Reeve JN (2011) Deletion of alternative pathways for reductant recycling in Thermococcus kodakarensis increases hydrogen production. Mol Microbiol 81:897-911. doi:10.1111/j.1365-2958.2011.07734.x

Sato T, Fukui T, Atomi H, Imanaka T (2003) Targeted gene disruption by homologous recombination in the hyperthermophilic archaeon Thermococcus kodakaraensis KOD1. J Bacteriol 185:210-220

Sato T, Imanaka H, Rashid N, Fukui T, Atomi H, Imanaka T (2004) Genetic evidence identifying the true gluconeogenic fructose1,6-bisphosphatase in Thermococcus kodakaraensis and other hyerthermophiles. J Bacteriol 186:5799-5807

Sato T, Fukui T, Atomi H, Imanaka T (2005) Improved and versatile transformation system allowing multiple genetic manipulations of the hyperthermophilic archaeon Thermococcus kodakaraensis. Appl Environ Microbiol 71:3889-3899

Soppa J (2013) Evolutionary advantages of polyploidy in halophilic archaea. Biochem Soc Trans 41:339-343

Takemasa R, Yokooji Y, Yamatsu A, Atomi H, Imanaka T (2011) Thermococcus kodakarensis as a host for gene expression and protein secretion. Appl Environ Microbiol 77:2392-2398. doi:10.1128/ aem.01005-10

Ventosa A, Oren A (1996) Halobacterium salinarum nom. corrig., a name to replace Halobacterium salinarium (Elazari-Volcani) and to include Halobacterium halobium and Halobacterium cutirubrum. Int J Syst Bacteriol 46:347. doi:10.1099/00207713-46-1-347

White MF, Bell SD (2002) Holding it together: chromatin in the Archaea. Trends Genet 18:621-626. doi:10.1016/ S0168-9525(02)02808-1

Yokooji Y, Sato T, Fujiwara S, Imanaka T, Atomi H (2013) Genetic examination of initial amino acid oxidation and glutamate catabolism in the Hyperthermophilic Archaeon Thermococcus kodakarensis. J Bacteriol 195:1940-1948. doi:10.1128/jb.01979-12

Zerulla K, Soppa J (2014) Polyploidy in haloarchaea: advantages for growth and survival. Frontiers in Microbiology 5:274. doi:10.3389/fmicb.2014.00274

Zerulla K, Chimileski S, Nather D, Gophna U, Papke RT, Soppa J (2014) DNA as a phosphate storage polymer and the alternative advantages of polyploidy for growth or survival. PLoS One 9:e94819. doi:10.1371/journal.pone.0094819 